\title{
Composite Adaptive Internal Model Control and Its Application to Boost Pressure Control of a Turbocharged Gasoline Engine
}

\author{
Zeng Qiu, Mario Santillo, Mrdjan Jankovic, Fellow, IEEE, and Jing Sun, Fellow, IEEE
}

\begin{abstract}
Internal model control (IMC) explicitly incorporates the plant model and its approximate inverse and offers an intuitive controller structure and calibration procedure. In the presence of plant-model uncertainty, combining the IMC structure with parameter estimation through the certainty equivalence principle leads to adaptive IMC (AIMC), where either the plant model or its inverse is identified. This paper proposes a composite AIMC (CAIMC) that explores the IMC structure and simultaneous plant dynamics and inverse dynamics identification to achieve improved performance of AIMC. A toy plant is used to illustrate the feasibility and potential of CAIMC. The advantages of CAIMC are later demonstrated on the boost-pressure control problem of a turbocharged gasoline engine. The design of the CAIMC assumes that the plant model and its inverse are represented by the first-order linear dynamics. The unmodeled dynamics and uncertainties due to linearization and variations in operating conditions are compensated through adaptation. The resulting CAIMC is first applied to a physics-based high-order and nonlinear proprietary turbocharged gasoline engine model, and then validated on a turbocharged $2-\mathrm{L}$ four-cylinder gasoline engine on a vehicle with vacuum-actuated wastegate. Both the simulation and experimental results show that the CAIMC cannot only effectively compensate for uncertainties but also auto-tune the IMC controller for the best performance.
\end{abstract}

Index Terms-Adaptive control, boost pressure control, calibration, internal model control (IMC), turbocharged gasoline engines.

\section{INTRODUCTION}

$\mathbf{I}$ NTERNAL MODEL CONTROL (IMC) is an intuitive control structure with simple tuning philosophy. It has found success in many applications in different industries, especially in the process industry [1]. As shown in Fig. 1, IMC incorporates the plant model as an explicit part of the IMC controller, and the feedback is acting on the difference between the plant and the model responses. When the model is the same

Manuscript received November 10, 2014; accepted February 15, 2015. Date of publication April 8, 2015; date of current version October 12, 2015. Manuscript received in final form March 13, 2015. This work was supported by Ford Motor Company, Dearborn, MI, USA. Recommended by Associate Editor H. Wang.

Z. Qiu is with the Department of Electrical Engineering and Computer Science, University of Michigan, Ann Arbor, MI 48109 USA (e-mail: connieqz@umich.edu).

M. Santillo and M. Jankovic are with Ford Motor Company, Dearborn, MI 48124 USA (e-mail: msantil3@ford.com; mjankov1@ford.com).

J. Sun is with the Department of Navel Architecture and Marine Engineering, University of Michigan, Ann Arbor, MI 48109 USA (e-mail: jingsun@umich.edu).

Color versions of one or more of the figures in this paper are available online at http://ieeexplore.ieee.org.

Digital Object Identifier 10.1109/TCST.2015.2414400

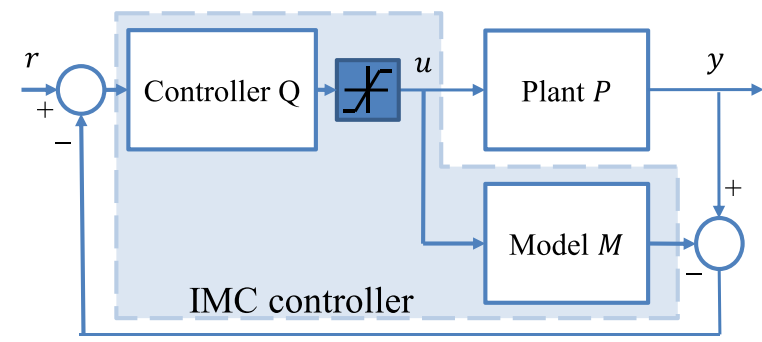

Fig. 1. IMC structure.

as the plant, the feedback will cease to have an effect and the structure will be equivalent to open-loop feedforward control. While the IMC works only for stable systems, there are several salient features that make it attractive, especially from the controller tuning point of view. For example, when the plant is stable and minimum phase, the controller $Q$ in Fig. 1 can be simply chosen as the inverse of the model. This inverse can be obtained by inverting the plant model $M$ and augmenting it with a low-pass filter, where the latter is used to assure causality of the controller and robustness of the closed-loop system. The time constant of the filter, as the only tuning parameter in this design, can be calibrated to achieve the desired bandwidth of the control system, thereby ensuring robustness.

The design, analysis, and implementation of IMC for linear systems have been well explored [1]. But for nonlinear systems, the results and tools for the IMC design and implementation are very limited. In particular, the online nonlinear inversion presents a great challenge due to high algorithmic and computational complexity. Economou et al. [2] proposed an IMC nonlinear dynamic inversion scheme for IMC implementation. Its derivation involves higher order derivatives, which may cause problems when noises and disturbances are present in the system. Treating a nonlinear model as a quasi-linear parameter-varying (LPV) model can help deriving the nonlinear inverse [3]. It is, however, shown that such a treatment works only in limited cases, and when it works, the derivation is computationally expensive and the subsequent design in the LPV framework is very demanding. For some specific nonlinear systems, the structure of the nonlinearity can be explored so that the inversion can be decomposed into cascaded inversions of several first-order systems [4], thereby alleviating the difficulty in high-order nonlinear system inversion. However, this is applicable only 
if the nonlinear system has the special structural property that lends itself for such a decomposition.

Another challenge in IMC design is in dealing with variations in the underlying plant and its operating environment. In this case, the IMC structure shown in Fig. 1 lends itself to certainty equivalence design that combines IMC with parameter estimation, resulting in an adaptive IMC (AIMC) [5]. Neural network, fuzzy logic, adaptive inverse control, and certainty equivalence principle with parameter estimation have all been exploited for AIMC. Hunt and Sbarbaro [6] and Nahas et al. [7] used artificial neural networks for AIMC, where the feasibility of simultaneous identification of the model and its inverse with neural networks was demonstrated. Boukezzoula et al. [8] and Xie and Rad [9] considered AIMC with fuzzy logic. A fuzzy dynamic model is proposed, and its inverse is derived from this model. For both neural networks and fuzzy logic, their black-box identification approach makes it difficult to incorporate physical knowledge about the plant in the IMC design. Shafiq [10] investigated AIMC with an adaptive inverse control strategy, in which the inverse is approximated by an adaptive finite-impulse response filter. A comprehensive study on AIMC with the certainty equivalence principle and parameter estimation has been carried out in [5], and many successful applications have been discussed in [11] and [12]. In this case, the standard certainty equivalence design approach is adopted, where the model is identified and the inverse is derived by inverting the estimated model. Stability, robustness, and performance issues are also addressed in [5].

For AIMC designed with the certainty equivalence approach, simultaneous identification of the model and the inverse is very tempting: IMC performs better with more accurate models and more accurate inverses. By identifying the inverse directly, as opposed to calculating the inverse model from the identified plant model, one can achieve a more accurate inverse dynamic model. Moreover, the direct identification of the inverse avoids online inversion of the identified model, which might be noninvertible under general excitation conditions. It also eliminates the need to design the filter in the inverse, thereby further simplifying the calibration process.

In this paper, we consider the AIMC design for systems with unknown and/or varying parameters. The variations of the parameters could be resulting from aging, or from changing operating conditions in nonlinear systems represented by linear models derived at specific operating conditions. A composite AIMC (CAIMC) is pursued, where the plant and inverse models are parameterized and identified simultaneously. This paper investigates the feasibility, performance, and advantages of CAIMC, first with a simple toy plant, then for a turbocharged gasoline engine model, and finally on a real plant. The identification is performed with the standard parametric models and the discrete-time projection algorithm, where other estimation algorithms can be easily incorporated if desired.

This paper is organized as follows. Section II provides an overview of the preliminaries: IMC and AIMC. Section III presents the CAIMC structure and its design procedures and validates it on an example problem. Section IV applies CAIMC on the boost control of a turbocharged gasoline engine model. The simulation results of the closedloop system using a high-order nonlinear proprietary model are presented. Section $\mathrm{V}$ is devoted to the experimental results, where the tests of the CAIMC on a vehicle are discussed and the effectiveness of the algorithm is demonstrated. Section VI draws the conclusion and summarizes this paper.

\section{BACKGROUND}

\section{A. Internal Model Control}

IMC is an intuitive control structure as shown in Fig. 1, where $P, M$, and $Q$ represent the plant, model, and controller, respectively. $M$ is an explicit part of the IMC controller. $Q$ takes the reference $r$ and the difference between $M$ and $P$ as its inputs. When the model is exact, i.e., $M=P$, the IMC structure becomes open loop. $Q$ can be designed such that the tracking objective is achieved optimally in $\mathrm{H}_{2}$ sense. Thus, one approach to IMC design is to solve the optimization problem

$$
\min _{Q}\|e\|_{2}=\min _{Q}\|(1-Q P) r\|_{2}
$$

subject to the constraint that $Q$ is stable and causal. The optimization (1) reaches the minimum at $Q=P^{-1}$, which is equivalent to $M^{-1}$ if the inverse exists and is feasible. For a stable and minimum phase plant, considering the constraints, $Q$ can be designed as $P^{-1}$ appended with a filter to make it causal.

IMC has many appealing properties [1], which can be extended to nonlinear systems [2], including the following.

1) Zero Offset: The controller will lead to zero steady-state error as long as the steady-state gain of $Q$ is the inverse of the steady-state gain of $M$.

2) Simple Tuning Philosophy: The only tuning parameter in IMC structure is the time constant of the filter augmented to the inverse. It reflects the bandwidth of the control system.

\section{B. AIMC With Certainty Equivalence Principle}

When there are uncertainties in the plants, AIMC combines identification with the IMC structure [5]. Most AIMC results found in the literature are based on the certainty equivalence principle, namely, the plant model was estimated, and then the estimated model $M$ was treated as the true one, based on which the AIMC was designed. That is, $Q$ was designed as in Section II-A by finding an approximate inverse of the estimated $M$. The structure is shown in Fig. 2 .

The identification of the plant can be performed with a standard parameter estimation approach. For nonlinear systems that can be approximated by a linear model at a given operating condition, an $n$ th-order linear dynamic model can be assumed to have the general form of [13]

$$
\begin{aligned}
& y^{(n)}+a_{n-1} y^{(n-1)}+\cdots+a_{0} y \\
& \quad=b_{n-1} u^{(n-1)}+b_{n-2} u^{(n-2)}+\cdots+b_{0} u
\end{aligned}
$$




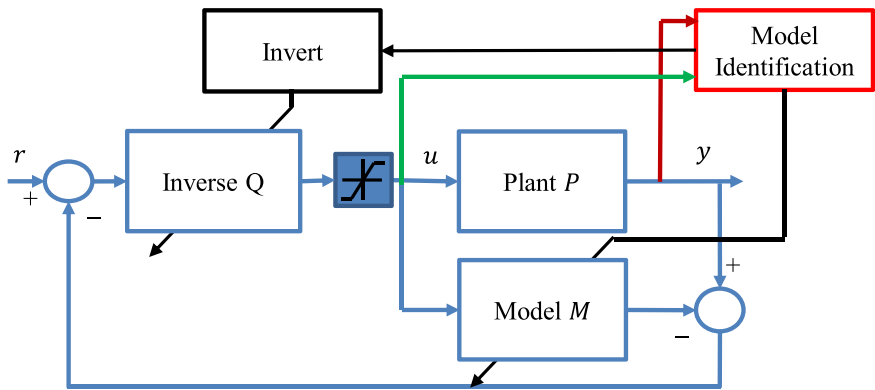

Fig. 2. Conventional AIMC structure.

from which one can obtain a linear parametric model

$$
z=\theta^{* T} \phi
$$

where the parameter vector

$$
\theta^{*}=\left[b_{n-1}, b_{n-2}, \ldots, b_{0}, a_{n-1}, a_{n-2}, \ldots, a_{0}\right]^{T}
$$

contains all the unknown parameters in (2). The signal $z$ and $\phi$ are defined as

$$
\begin{aligned}
z= & \left\{\frac{s^{n}}{\Lambda(s)}\right\} y \\
\phi= & {\left[\left\{\frac{s^{n-1}}{\Lambda(s)}\right\} u,\left\{\frac{s^{n-2}}{\Lambda(s)}\right\} u, \ldots,\left\{\frac{1}{\Lambda(s)}\right\} u,-\left\{\frac{s^{n-1}}{\Lambda(s)}\right\} y\right.} \\
& \left.-\left\{\frac{s^{n-2}}{\Lambda(s)}\right\} y, \ldots,-\left\{\frac{1}{\Lambda(s)}\right\} y\right]^{T}
\end{aligned}
$$

where $z$ and $\phi$ are referred to as the observation and the regressor, respectively. $\{\cdot\}$ represents the dynamic operators, whose transfer function is $(\cdot) . \Lambda(s)=s^{n}+$ $\lambda_{n-1} s^{n-1}+\cdots+\lambda_{0}$ is chosen as a Hurwitz polynomial, $1 / \Lambda(s)$ is a filter that is introduced to avoid using derivatives in parameter estimation [13]. $\theta^{*}$ can be estimated using identification algorithms, such as the least-squares algorithm, gradient method, and projection algorithm, based on the input-output data. If discrete-time adaptation is used, then the updates of $\theta(i)$ at the $i$ th sample will take the general form of

$$
\theta(i)=\theta(i-1)+f(\phi(i), \epsilon(i), \phi(i-1), \ldots)
$$

where $\epsilon(i)=z(i)-\theta^{T}(i-1) \phi(i)$ is the prediction error and $f$ is the correction term. For example, if the projection algorithm [13] is used, then (7) has the form

$$
\theta(i)=\theta(i-1)+\frac{\gamma \phi(i)}{\sigma+\phi(i)^{T} \phi(i)} \epsilon(i)
$$

where $0<\gamma<2$ and $\sigma>0$. A deadzone is often applied to $\epsilon(i)$ for robustness [13].

\section{Composite Adaptive Internal Model Control}

CAIMC incorporates simultaneous identification of the model and the inverse of the plant, as shown in Fig. 3. The motivation for the simultaneous identification is that the IMC performs better with a more accurate plant model and a more accurate plant inverse, which can be achieved by simultaneous identification. Another incentive for identifying the inverse directly is that it avoids numerical inversion.

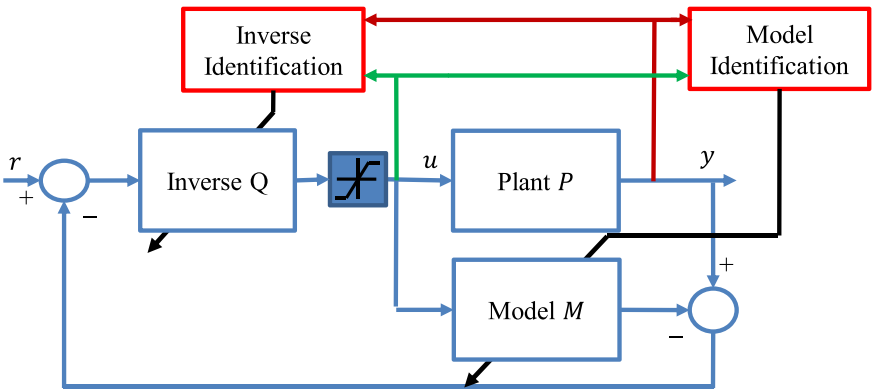

Fig. 3. CAIMC structure.

For CAIMC, one parametric model is chosen for plant dynamics identification, which follows the discussion in Section II-B. Another parametric model is used for the purpose of inverse dynamics identification, which can be treated similarly by reversing the role of the input and the output in the model representation. Therefore, the design procedure of CAIMC, following the certainty equivalence principle, can be described as follows.

1) Formulate a model and an inverse model structure based on the plant dynamics, i.e., define the order of the plant model and inverse model, while taking the complexity into consideration.

2) Derive the parametric models of the proposed model structures, and identify the unknown parameters using an appropriate identification algorithm.

3) Embed the identified model and inverse into the IMC structure.

To demonstrate the benefits of CAIMC and further clarify the design procedures that we proposed, CAIMC is applied to a simple example before it is used for a more elaborate engineering application. Consider the control problem of a nonlinear system

$$
\dot{x}=-x^{2}+(x+c) u, \quad y=x+u
$$

and $c=1$ is used in the following simulations. The control objective is that $y$ tracks a given reference $r$. Note that the nonlinear plant has a direct path from the input to the output (i.e., its relative degree is 0 ), and its steady state depends on the parameter $c$. We assume that the parameter $c$, as well as the exact form of the nonlinear dynamics (including its relative degree), is unknown. Therefore, when we use a linear model to approximate this nonlinear system, the model and the inverse will take the form of $k /(\tau s+1)$ and its inverse $(1 / k)(b s+1) /(a s+1)$. The parameters $\tau, k, a$, and $b$ in this linear model will depend on the plant uncertainty, as well as the reference input $r$, which defines the linearization point.

1) Parametric Models and Identification: Considering the plant dynamics, we proposed the model and inverse structure of the form $k /(\tau s+1)$ and $(1 / k)(b s+1) /(a s+1)$, respectively, for simultaneous identification, in which $\tau, k$, $a$, and $b$ are unknown. Following the procedure described in Section II-B, the parametric representations for the model and the inverse can be obtained. For the plant identification, 
TABLE I

PARAMETERS FOR TOY PROBLEM SIMULATION

\begin{tabular}{|c|c|c|c|c|c|c|c|}
\hline Identification & $\lambda$ & $\theta(0)$ & & $\gamma$ & & $\sigma$ & deadzone \\
\hline Plant dynamics & 10 & $\begin{array}{c}0.5 \\
1\end{array}$ & & $\begin{array}{c}0.5 \\
0\end{array}$ & $\left.\begin{array}{l}0 \\
1\end{array}\right]$ & 0.5 & 0.5 \\
\hline Inverse dynamics & 10 & $\begin{array}{c}0.5 \\
1 \\
0.5\end{array}$ & $\begin{array}{l}1 \\
0 \\
0\end{array}$ & $\begin{array}{c}0 \\
0.1 \\
0\end{array}$ & $\begin{array}{c}0 \\
0 \\
0.1\end{array}$ & 2 & 0.2 \\
\hline
\end{tabular}

the parametric model and the associated signals are defined as

$$
\begin{aligned}
z_{1} & =\theta_{1}^{* T} \phi_{1} \\
z_{1} & =\left\{\frac{1}{\Lambda_{1}(s)}\right\} y, \quad \theta_{1}^{*}=[\tau, k]^{T} \\
\phi_{1} & =\left[-\left\{\frac{s}{\Lambda_{1}(s)}\right\} y,\left\{\frac{1}{\Lambda_{1}(s)}\right\} u\right]^{T} \\
\Lambda_{1}(s) & =s+\lambda_{1} .
\end{aligned}
$$

For the inverse identification, the parametric model and the associated signals are defined as

$$
\begin{aligned}
z_{2} & =\theta_{2}^{* T} \phi_{2} \\
z_{2} & =\left\{\frac{1}{\Lambda_{2}(s)}\right\} u, \quad \theta_{2}^{*}=\left[\frac{b}{k}, \frac{1}{k}, a\right]^{T} \\
\phi_{2} & =\left[\left\{\frac{s}{\Lambda_{2}(s)}\right\} y,\left\{\frac{1}{\Lambda_{2}(s)}\right\} y-\left\{\frac{s}{\Lambda_{2}(s)}\right\} u\right]^{T} \\
\Lambda_{2}(s) & =s+\lambda_{2}
\end{aligned}
$$

where $\lambda_{1}$ and $\lambda_{2}$ are the filter parameters. With the discretetime projection algorithm (8), the unknown parameters can be identified.

2) IMC Result: Two AIMC schemes are implemented and compared. One is the conventional AIMC as in Fig. 2 designed with the certainty equivalence principle, where the plant is identified and the inverse is calculated based on the identified model using the frozen parameter approach. A low-pass filter whose time constant is represented by $\tau_{i}$ is added to the inverse to assure causality. The other is CAIMC, where the plant dynamics and inverse dynamics are identified simultaneously and no online calculation is involved for model inversion. The projection algorithm in (8) with deadzone is adopted for parameter estimation of $\theta_{1}^{*}$ and $\theta_{2}^{*}$ for its simplicity and robustness. Based on property 1) in Section II-A, zero steady-state tracking can be achieved by keeping a consistent $k$ in the plant and inverse models. We simply used the estimated $k$ in $\theta_{1}$ in both the model and the inverse. The parameters used in the simulation are summarized in Table I.

A square wave of amplitude 0.5 and period $18 \mathrm{~s}$ is used for the reference $r$. A white noise measurement of $3 \%$ is added to the input and output measurements in all simulations. The simulation results are shown in Fig. 4 for a time interval of $[142,178]$ s. Several conventional AIMC results are shown with different $\tau_{i}$. Before $142 \mathrm{~s}$, the parameters have not converged yet. One can observe that CAIMC and AIMC based on the first-order linear model can handle the nonlinear plant, even with a different relative degree. Moreover, CAIMC achieves the performance that is as good as that is achieved with AIMC, without the need to tune the filter parameter $\tau_{i}$.
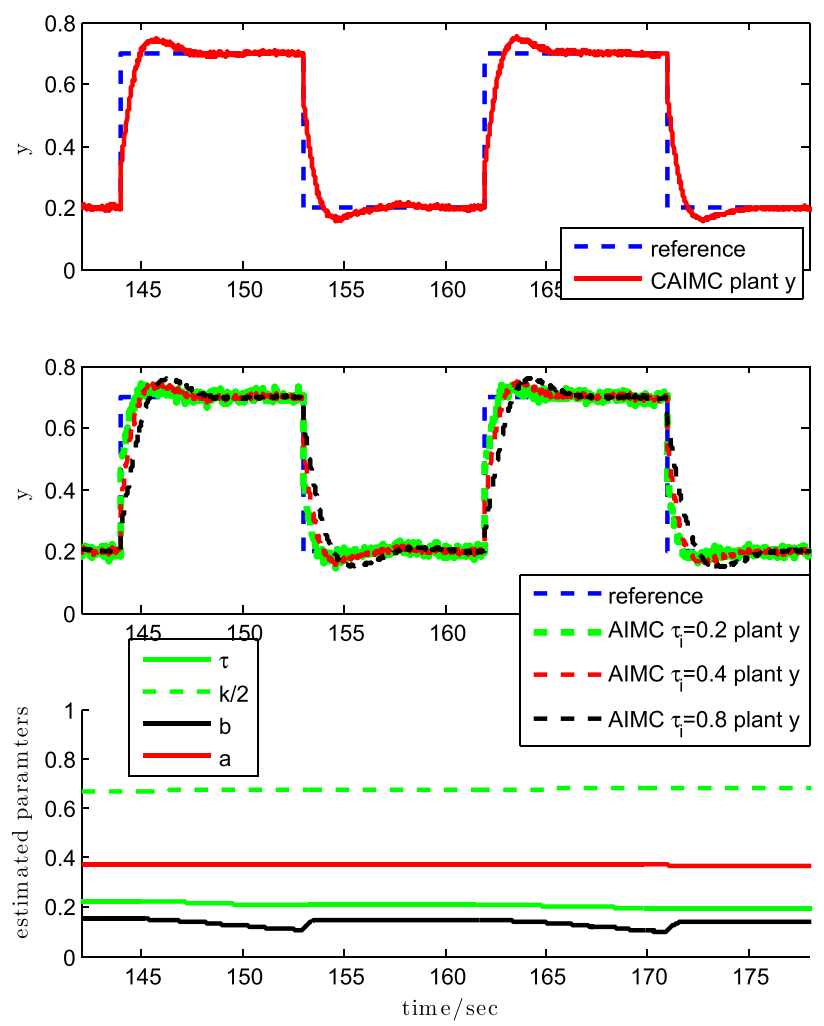

Fig. 4. Comparison of CAIMC and conventional AIMC for the toy problem (9).

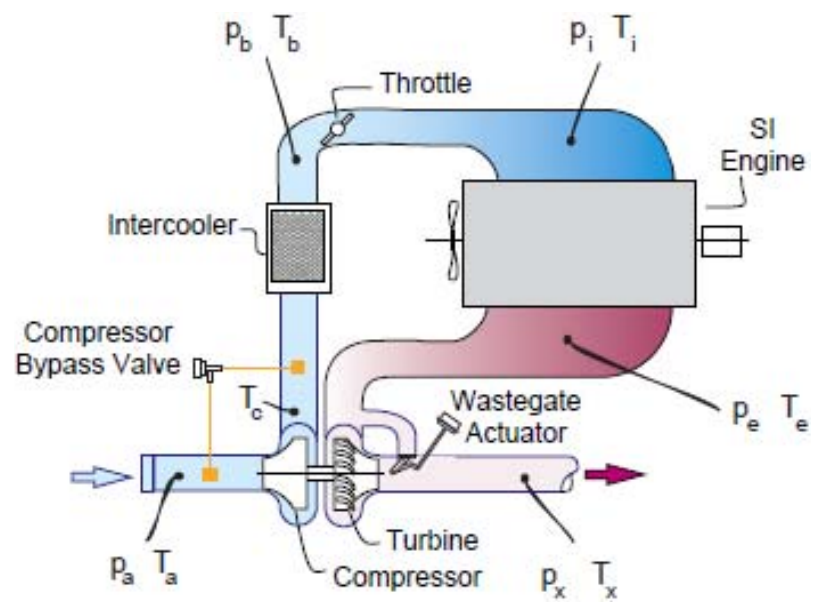

Fig. 5. Turbocharged gasoline engine structure [14].

\section{Application to Boost Pressure Control of A TURBOCHARGED GASOLINE ENGINE}

Nowadays, gasoline engines are aggressively downsized for fuel economy. To meet the customer demand for engine power on the downsized engine, turbochargers are adopted to increase the density of the air, thereby increasing the engine power. Boost control is a critical technology to fully exploit the advantage of the turbocharged gasoline engine.

Fig. 5 shows the schematic of a turbocharged gasoline engine. The compressor and the turbine are connected through a mechanical link. Air is taken in by the compressor, goes through the cooler and throttle, and then it is pushed into the cylinders. The exhaust has two outlets: the turbine and the 


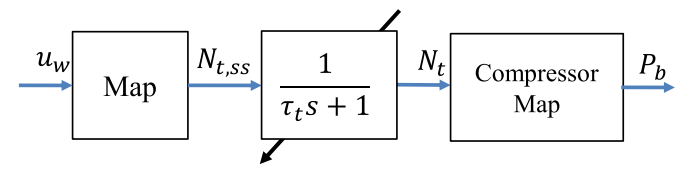

Fig. 6. Simplified first-order model structure [15].

(a)

(b)

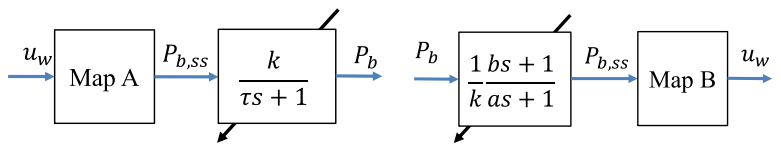

Fig. 7. Plant and inverse dynamic models. (a) First-order plant-model structure for identification. (b) First-order inverse-model structure for identification.

pathway controlled by a wastegate. By changing the wastegate opening, the turbo airflow can be changed, which affects the turbo/compressor speed and changes the boost pressure. The boost control problem is to use the wastegate as the actuator to control the boost pressure to its reference. A physics-based high-order nonlinear proprietary turbocharged gasoline engine model serves as the plant in the following design.

This application is motivated by the successful industrial applications of IMC and the need for developing robust and easy-to-calibrate powertrain control solutions. The turbocharged gasoline engine is a high-order nonlinear system, which operates in different environments. By adding identification, the AIMC structure can handle the uncertainties including the unmodeled dynamics and the varying operating environments. The direct identification of the inverse dynamics in CAIMC will allow a better approximation of the inverse of the plant, making it attractive for the boost-pressure control problem.

\section{A. Plant and Inverse Dynamic Models}

CAIMC involves plant dynamics and inverse dynamics identification, whose first step is to find feasible model and inverse structures to capture these dynamics. To sufficiently describe the air dynamics of a turbocharged gasoline engine, a fourthorder nonlinear first principle model is often derived [4]. Karnik and Jankovic [15] simplified the fourth-order nonlinear model into a model as shown in Fig. 6. It includes a steadystate mapping from the wastegate $u_{w}$ to the turbo speed $N_{t}$, a first-order linear model whose time constant is gain scheduled, and a compressor mapping. This structure motivates us to further simplify and propose a model structure as in Fig. 7(a), which combines the two mappings into a steady-state mapping from the wastegate $u_{w}$ to the boost pressure $P_{b}$ and eliminates the intermediate variable of the turbo speed $N_{t}$. The corresponding plant inverse model structure is shown in Fig. 7(b).

\section{B. Plant and Inverse Model Identification}

Following the procedure outlined in Section II-B, the parametric models for the plant and the inverse dynamics are obtained. Let $u=P_{b, \mathrm{ss}}$ and $y=P_{b}$. For the plant identification, the parametric model is $z_{1}=\theta_{1}^{* T} \phi_{1}$ with the same structure as (10). For the inverse dynamic model identification, the parametric model is $z_{2}=\theta_{2}^{* T} \phi_{2}$ with the (a)

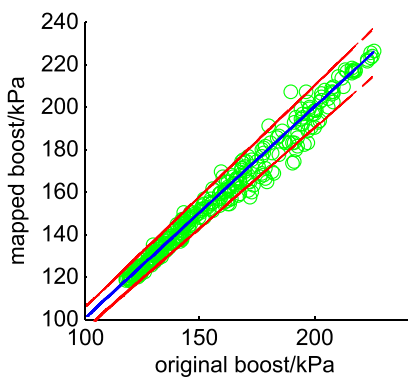

(b)

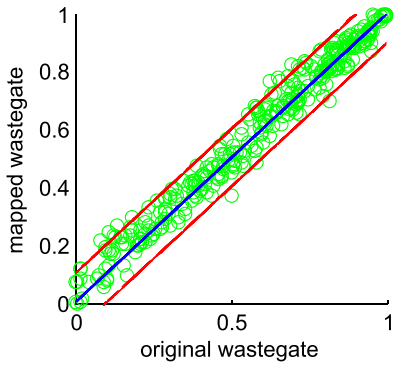

Fig. 8. Mapping accuracy. (a) From $u_{w}$ to $P_{b}$. (b) From $P_{b}$ to $u_{w}$. Black line represents exact fit. In (a), red line represents $\pm 5 \%$ error. In (b), red line represents \pm 0.1 error.

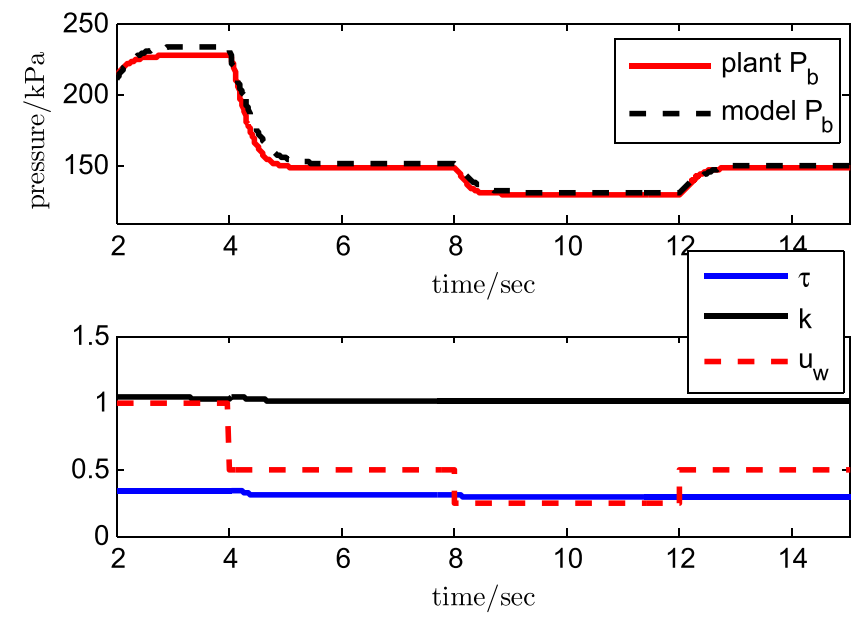

Fig. 9. Turbocharged gasoline engine model identification.

same structure as (11). The identification algorithm used here is the projection algorithm with deadzone, chosen for its simplicity and robustness.

\section{Derivation of the Static Maps}

The model and inverse structures in Fig. 7(a) and (b) require a steady-state mapping between $P_{b}$ and $u_{w}$. As the throttle opening and the engine speed change, this mapping changes. A significant variable that affects the mapping is the engine airflow, because the engine airflow and $u_{w}$ determine the turbo airflow, which determines the turbo/compressor speed, therefore affecting $P_{b}$ significantly. We formulate a lookup table that depends on the engine airflow between the steady-state values of $P_{b}$ and $u_{w}$. For wastegate $u_{w}$, 0 means open and 1 means closed. The accuracy of the mapping by the lookup table is shown in Fig. 8(a) and (b). As shown in Fig. 8(a), mapping from $u_{w}$ to $P_{b}$ is mostly within $\pm 5 \%$ error. As shown in Fig. 8(b), mapping from $P_{b}$ to $u_{w}$ is mostly within \pm 0.1 . A gain $k$ is added to the model and inverse structure in Fig. 7(a) and (b) to compensate for the mapping inaccuracy.

\section{Simulation Results}

With the discrete-time projection algorithm and the static maps, which are derived offline, the plant and the inverse dynamics are identified. Fig. 9 shows a comparison of the responses of the plant, which is a high-order Ford proprietary 

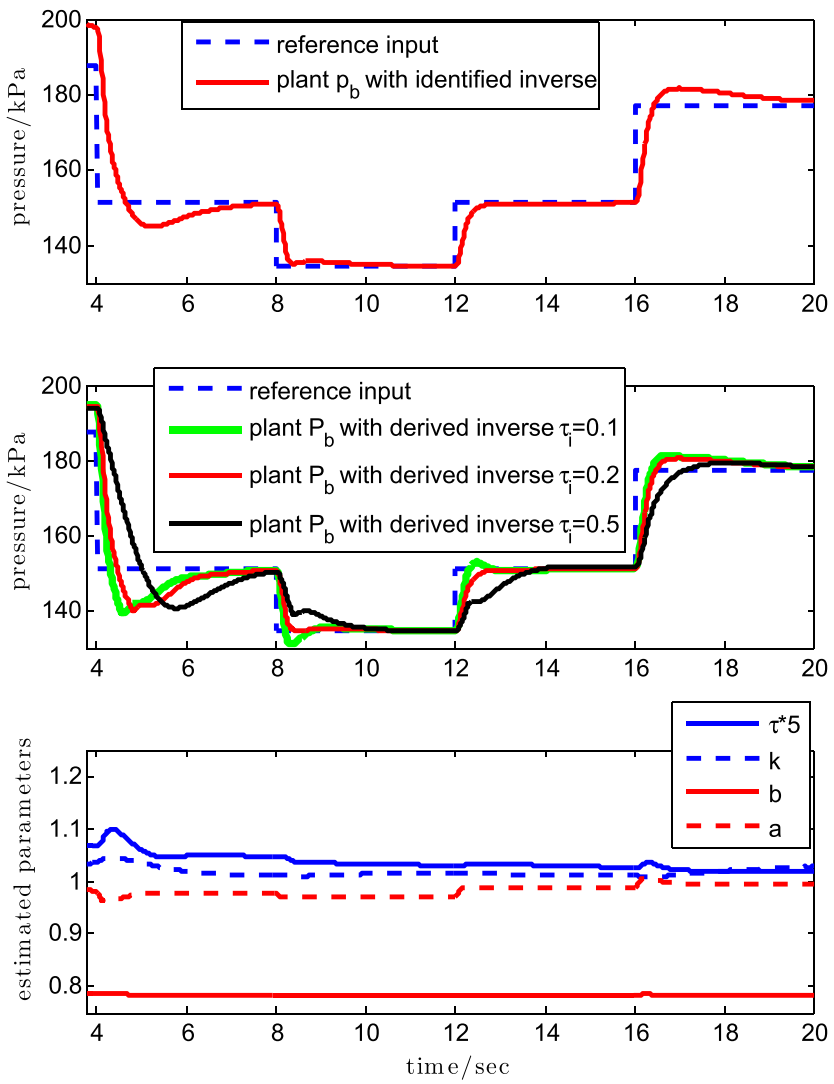

Fig. 10. Turbocharged gasoline engine inverse identification.

model, and the identified first-order model. One can observe that the two responses are very close. An inverse model can be derived by inverting this model. To validate the inverse model, we feed a reference input to the inverse, whose output then feeds into the plant. This validation is performed for both the inverse model derived from the estimated plant model and the one identified directly. Fig. 10 shows the results. The identified inverse performs as well as the best derived inverse in terms of approximating the plant inverse. Although steady-state error exists in both the plant and inverse identification results due to mapping inaccuracy, the steady-state tracking problem can be easily tackled in the IMC structure based on property 1) in Section II-A, by keeping a consistent $k$ in the plant and inverse models. We simply used the estimated $k$ in $\theta_{1}^{*}$ in both the model and the inverse. This paper verifies that the first-order model and inverse structures in Fig. 7(a) and (b) are feasible for representing the plant and its inverse dynamics.

Fig. 11 shows the implementation schematic of the CAIMC, where the first-order model and inverse model structures given in Fig. 7(a) and (b) are incorporated. Two AIMC schemes are implemented and compared: 1) CAIMC as shown in Fig. 11 and 2) the conventional AIMC as shown in Fig. 2, in which the model structure is as in Fig. 7(a). The parameters used in simulations are summarized in Table II. In conventional AIMC, the time constant of the filter that is appended to the inverse is carefully tuned to achieve the best performance. For CAIMC, however, such a tuning is not needed. The CAIMC results are shown in Fig. 12, while the

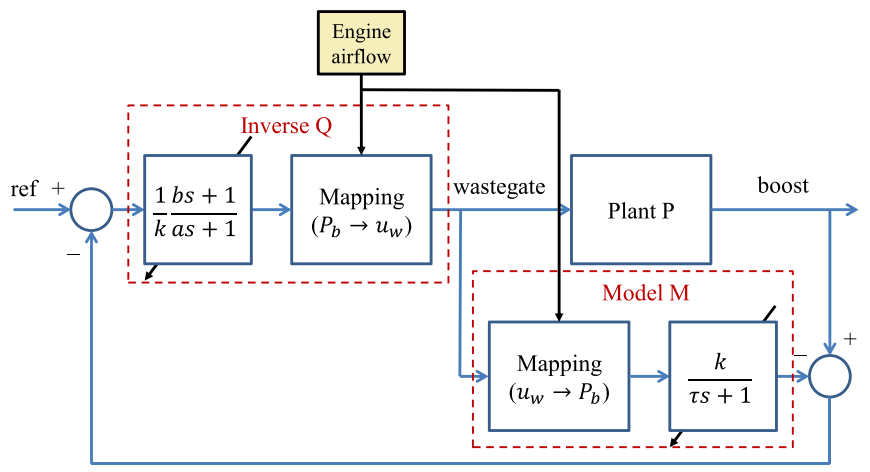

Fig. 11. CAIMC structure with the proposed model and inverse for the turbocharged gasoline engine.

TABLE II

Parameters of the Turbocharged Gasoline ENGine Model Simulation

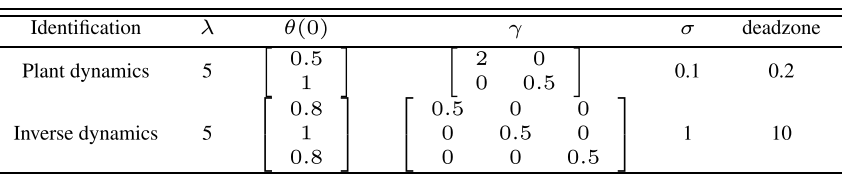
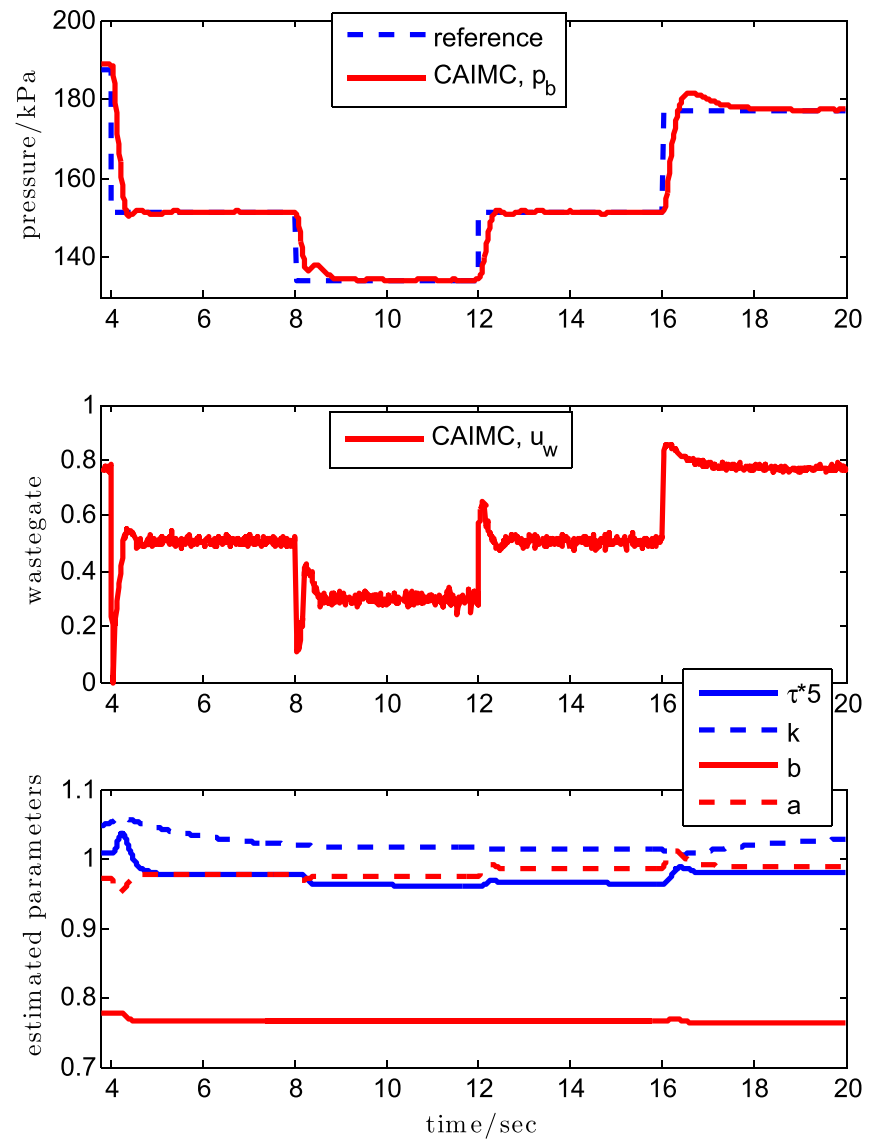

Fig. 12. CAIMC simulation result for the turbocharged gasoline engine.

conventional AIMC results are shown in Fig. 13, in which several results are shown with different $\tau_{i}$. One can observe that the performance of CAIMC matches the best performance 


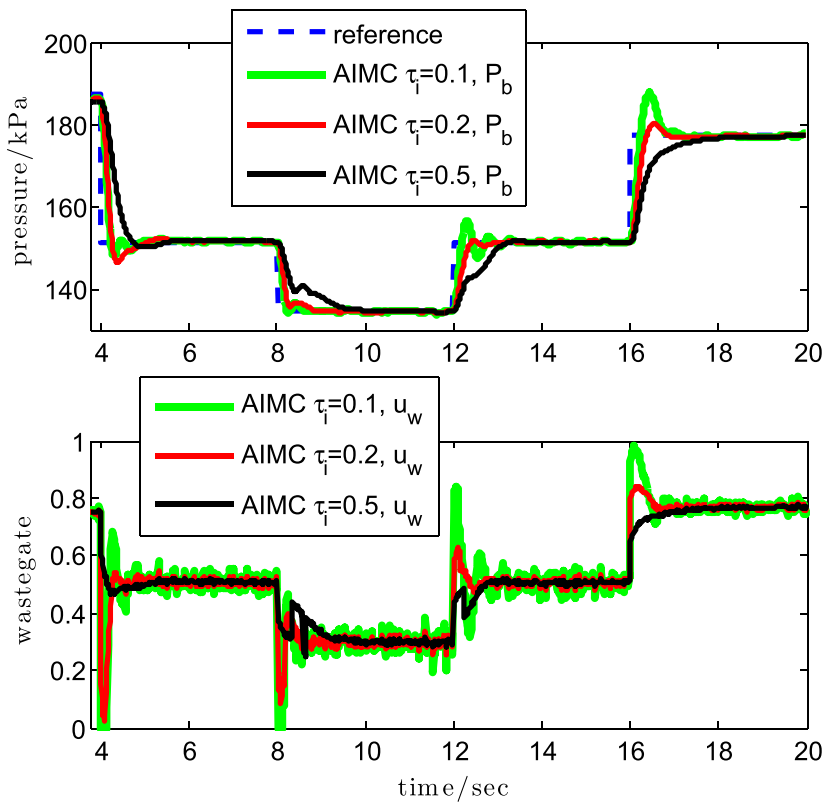

Fig. 13. Conventional AIMC simulation result for the turbocharged gasoline engine.

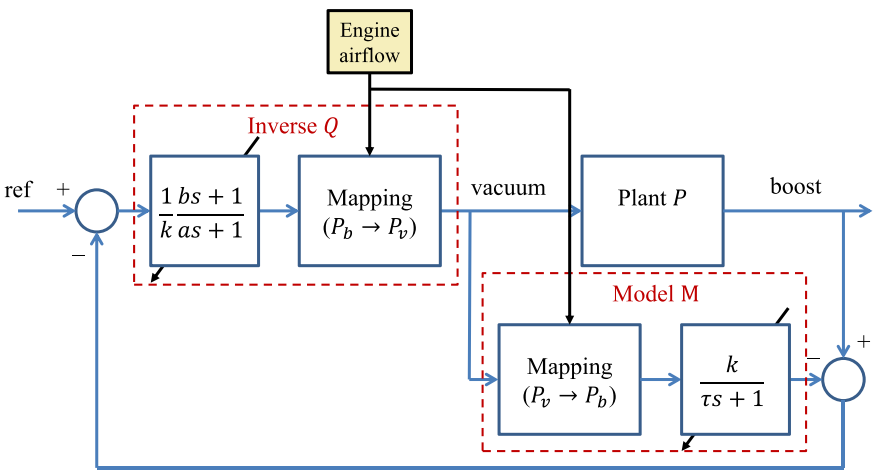

Fig. 14. CAIMC structure with the proposed model and inverse for the turbocharged gasoline engine in-vehicle testing.

of AIMC, thereby demonstrating the auto-tuning capability of the proposed CAIMC. Note that the parameters have not converged yet before $2 \mathrm{~s}$ in Fig. 9 and before $4 \mathrm{~s}$ in Figs. 10, 12, and 13.

\section{Vehicle Experimental VALIDATION}

The experiments were performed on a vehicle (Ford Explorer EcoBoost) with a turbocharged four-cylinder 2-L gasoline engine with vacuum-actuated wastegate. The vacuum in the wastegate canister determines the position of the wastegate. In this case, lower vacuum corresponds to more open wastegate and higher vacuum to more closed wastegate. The control objective is to use the wastegate canister vacuum as the actuator to control the boost pressure $P_{b}$ to follow its reference. The schematic of the strategy is shown in Fig. 14, which is very similar to the control structure for the computer simulation in Fig. 11, except that the actuator is the wastegate canister vacuum $P_{v}$ instead of the wastegate position. Note that the dynamics from $P_{v}$ to the wastegate position are very

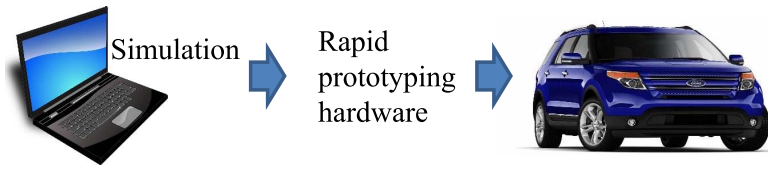

Fig. 15. Rapid control prototyping process.

fast, therefore it is reasonable to keep the first-order model and inverse structure proposed in Fig. 7. To accommodate the actuator of wastegate canister vacuum $P_{v}$, the model $M$ includes a mapping from $P_{v}$ to the boost pressure $P_{b}$, and the inverse $Q$ includes a mapping from $P_{b}$ to $P_{v}$. Both mappings were analyzed and shown to be within $\pm 8 \%$ error. The parametric models for identification are exactly the same as those used in Section IV for the design in (10) and (11).

\section{A. Experimental Setup and Validation Approach}

For experiments, the rapid control prototyping tools as in Fig. 15 were adopted: the boost control strategy was coded in MATLAB/Simulink, ${ }^{1}$ and then compiled and executed in real time on the hardware platform dSPACE. ${ }^{2}$ dSPACE can communicate with powertrain control module of the vehicle to access vehicle data and command wastegate canister vacuum. The boost control strategy was executed at a sample time of $15 \mathrm{~ms}$. Both the boost pressure and the wastegate canister pressure were measured by pressure sensors. An accurate estimation of the engine airflow was accessible. The identifications of $M$ and $Q$ were simultaneously performed online. The identified parameters $\tau, k, a$, and $b$ in $M$ and $Q$ were updated at each sample time.

Three different control algorithms were tested:

1) the baseline controller: a well-tuned PI controller with antiwindup and extensive gain-scheduled feedforward and feedback control;

2) CAIMC as shown in Fig. 14;

3) the conventional AIMC as shown in Fig. 2 with a welltuned time constant of the filter that is appended to the inverse.

The experiments were performed by driving the vehicle on the Ford test track in Dearborn, Michigan. Due to the difference in the driver behavior, the operating points and boost pressure references vary for each test, which presents challenges for test repeatability. To minimize the variation, we designed the following test procedure.

1) Start from a low vehicle speed and press the gas pedal to the ground such that the throttle is wide open.

2) Maintain the vehicle speed at $45 \mathrm{mi} / \mathrm{h}$ and enable the cruise control that sets the vehicle speed at $70 \mathrm{mi} / \mathrm{h}$.

3) Repeat the last two steps.

\section{B. Experimental Results}

With the experimental setup and test procedure proposed, the results of different control algorithms are presented and compared. For the baseline controller, when the airflow is

\footnotetext{
${ }^{1}$ MATLAB and Simulink are registered trademarks of The MathWorks, Inc. ${ }^{2} \mathrm{dSPACE}$ is a registered trademark of dSPACE GmbH.
} 
TABLE III

PARAMETERS OF TURBOCHARGED GASOLINE ENGINE VEHICLE TESTING

\begin{tabular}{|c|c|c|c|c|c|c|c|}
\hline Identification & $\lambda$ & $\theta(0)$ & & $\gamma$ & & $\sigma$ & deadzone \\
\hline Plant dynamics & 3.3 & $\begin{array}{c}0.2 \\
1\end{array}$ & & $\begin{array}{cc}5 & 0 \\
0 & 0.5\end{array}$ & & 0.1 & 3 \\
\hline Inverse dynamics & 3.3 & $\begin{array}{c}0.3 \\
1 \\
0.5\end{array}$ & $\begin{array}{l}5 \\
0 \\
0\end{array}$ & $\begin{array}{c}0 \\
0.5 \\
0\end{array}$ & $\begin{array}{l}0 \\
0 \\
0.5\end{array}$ & 0.1 & 10 \\
\hline
\end{tabular}
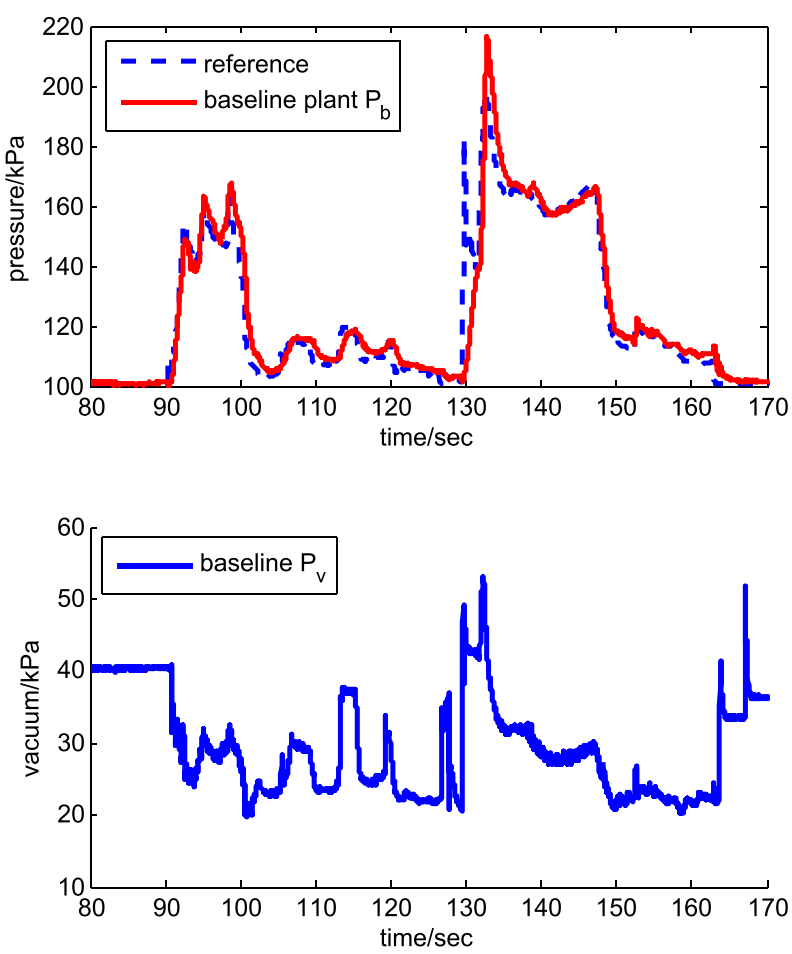

Fig. 16. Baseline controller vehicle testing result.

low, the default vacuum is $40.6 \mathrm{kPa}$. To make a fair comparison, the same default vacuum is applied to the other control algorithms. For CAIMC and conventional AIMC vehicle testing, the parameters are given in Table III. The same parameters and initial conditions are used in CAIMC and conventional AIMC whenever applicable.

Figs. 16-18 show the vehicle testing results of the baseline controller, CAIMC, and a well-tuned AIMC controller, respectively. For all the tests, there were a portion when the throttle is wide open $(90-100 \mathrm{~s}$ in Fig. 16, 105-115 s in Fig. 17, and 105-115 s in Fig. 18) and another portion when the vehicle is running in cruise control (130-160 s in Fig. 16, 145-175 $\mathrm{s}$ in Fig. 17, and 145-175 s in Fig. 18).

All the tests have comparable results; however, the baseline (Fig. 16) is achieved with sophisticated gain scheduling and extensive calibration. The conventional AIMC (Fig. 18) is achieved with the filter parameter that is tuned on the simulation model, and then retuned on the vehicle. The CAIMC (Fig. 17) achieved the performance without any vehicle tuning. To show the effect of adaptation, the performance of
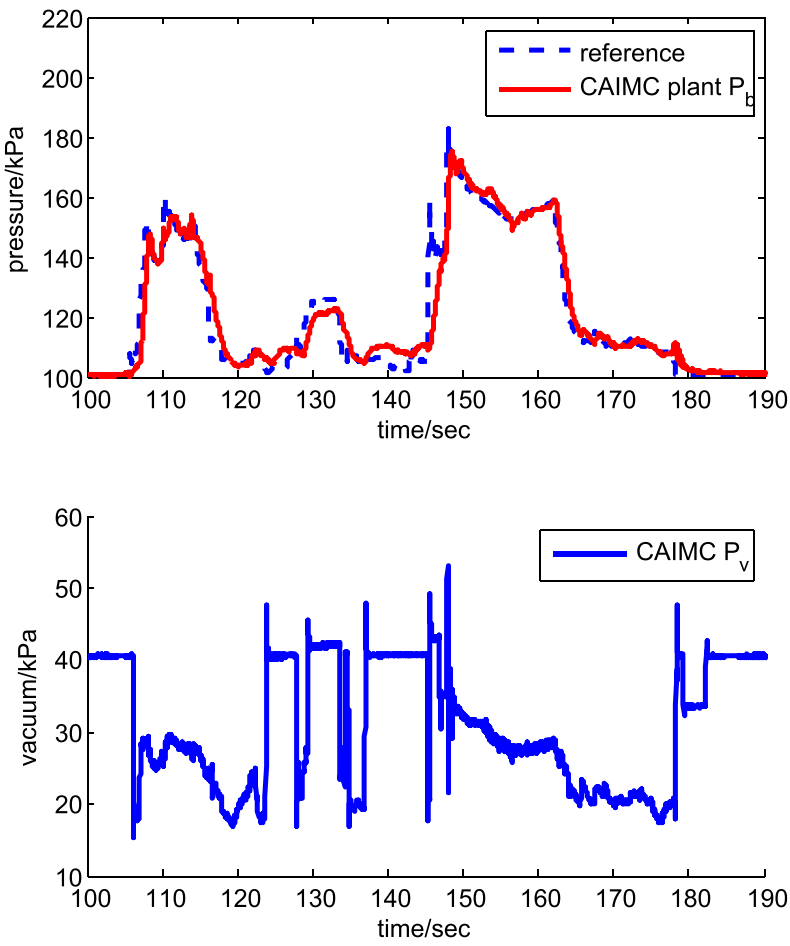

Fig. 17. CAIMC vehicle testing result.
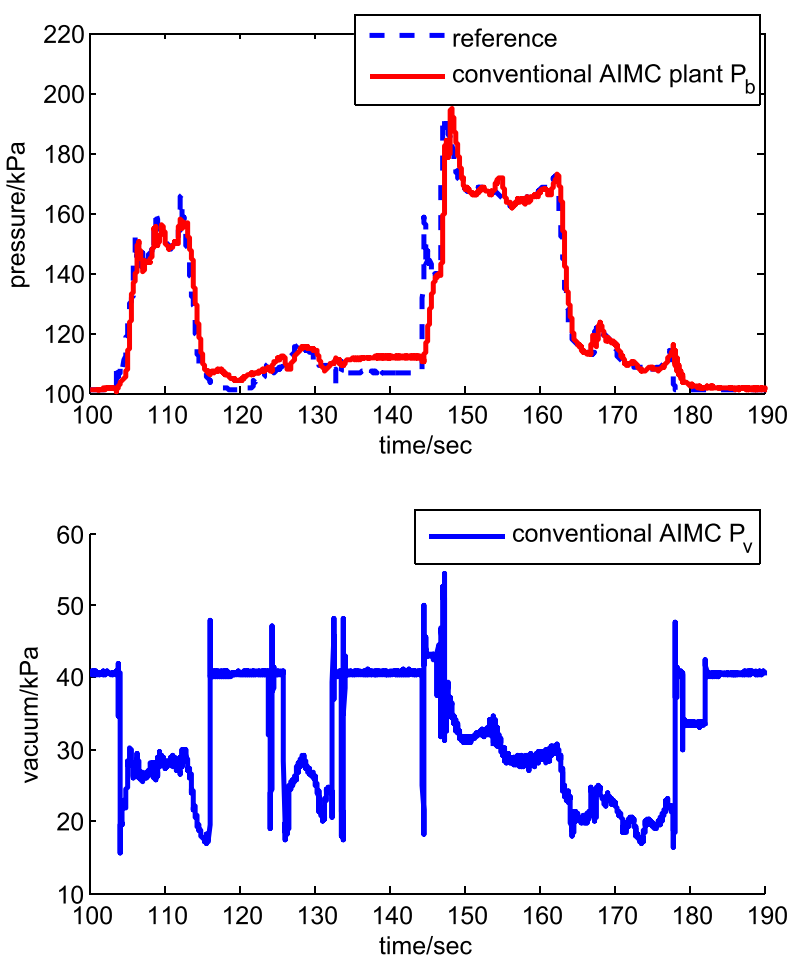

Fig. 18. Conventional AIMC vehicle testing result.

the system before parameter convergence was also evaluated as in Fig. 19.

The identified parameters are shown in Fig. 20. The parameters have not converged for $40 \mathrm{~s}$, which explains the bad control performance before $40 \mathrm{~s}$ and demonstrates the benefits of adaptation. 

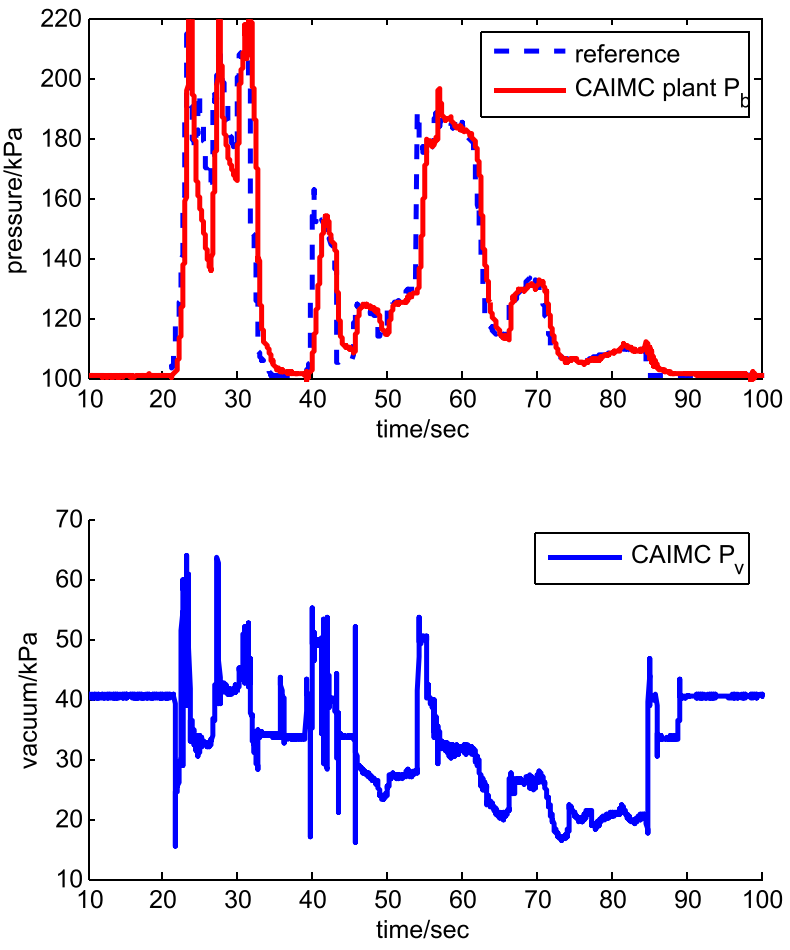

Fig. 19. CAIMC vehicle testing result before parameter convergence.

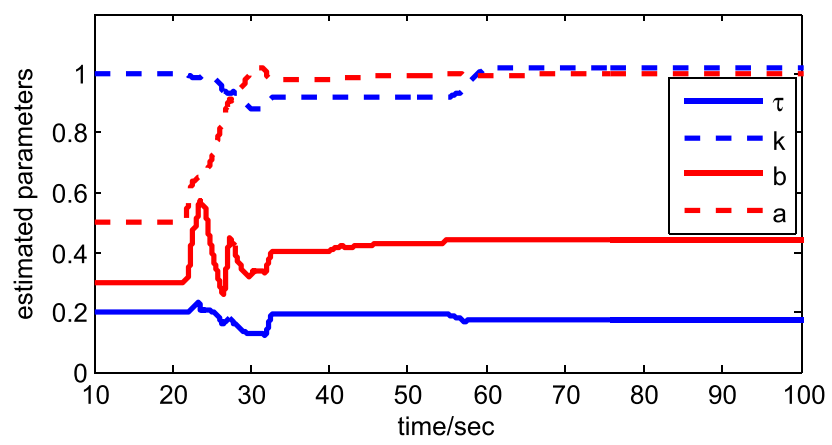

Fig. 20. CAIMC vehicle testing identified parameters.

\section{CONCLUSION}

CAIMC that combines the IMC structure with simultaneous identification of the plant and inverse models is proposed. Its benefits are demonstrated, using an example of controlling a simple nonlinear system. The results are compared with those of the conventional AIMC, where only the model identification is performed and the inverse is numerically derived from the identified model. The design procedure is summarized. The CAIMC is applied to the nonlinear boost-pressure control problem of a turbocharged gasoline engine. Numerical simulations and experiments show advantages, particularly, in terms of tuning effort required, compared with the AIMC with only plant model identification. Future work in this direction could include establishing general stability and performance for the CAIMC systems and developing more comprehensive design guidelines for exploring the simultaneous identification.

\section{ACKNOWLEDGMENT}

The authors would like to thank Dr. A. Karnik, M. Uhrich, and Dr. H. Ossareh from the Ford Motor Company for helping with software strategy, simulation, and vehicle setup.

\section{REFERENCES}

[1] M. Morari and E. Zafiriou, Robust Process Control. Upper Saddle River, NJ, USA: Prentice-Hall, 1989.

[2] C. G. Economou, M. Morari, and B. O. Palsson, "Internal model control: Extension to nonlinear system," Ind. Eng. Chem. Process Design Develop., vol. 25, no. 2, pp. 403-411, Apr. 1986.

[3] J. Mohammadpour, J. Sun, A. Karnik, and M. Jankovic, "Internal model control design for linear parameter varying systems," in Proc. Amer. Control Conf. (ACC), Jun. 2013, pp. 2409-2414.

[4] Z. Qiu, J. Sun, M. Jankovic, and M. Santillo, "Nonlinear internal model controller design for wastegate control of a turbocharged gasoline engine," in Proc. Amer. Control Conf. (ACC), Jun. 2014, pp. 214-219.

[5] A. Datta, Adaptive Internal Model Control. New York, NY, USA: Springer-Verlag, 1998.

[6] K. J. Hunt and D. Sbarbaro, "Neural networks for nonlinear internal model control," IEE Proc. D, Control Theory Appl., vol. 138, no. 5, pp. 431-438, Sep. 1991.

[7] E. P. Nahas, M. A. Henson, and D. E. Seborg, "Nonlinear internal model control strategy for neural network models," Comput. Chem. Eng., vol. 16, no. 12, pp. 1039-1057, Dec. 1992.

[8] R. Boukezzoula, S. Galichet, and L. Foulloy, "Fuzzy nonlinear adaptive internal model control," Eur. J. Control, vol. 7, no. 5, pp. 541-556, 2001.

[9] W. F. Xie and A. B. Rad, "Fuzzy adaptive internal model control," IEEE Trans. Ind. Electron., vol. 47, no. 1, pp. 193-202, Feb. 2000.

[10] M. Shafiq, "Internal model control structure using adaptive inverse control strategy," ISA Trans., vol. 44, no. 3, pp. 353-362, Jul. 2005.

[11] D. Rupp and L. Guzzella, "Adaptive internal model control with application to fueling control," Control Eng. Pract., vol. 18, no. 8, pp. 873-881, Aug. 2010.

[12] N. R. L. Narayanan, P. R. Krishnaswamy, and G. P. Rangaiah, "An adaptive internal model control strategy for pH neutralization," Chem. Eng. Sci., vol. 52, no. 18, pp. 3067-3074, Sep. 1997.

[13] P. Ioannou and J. Sun, Robust Adaptive Control. New York, NY, USA: Dover, 2012.

[14] J. H. Buckland, "Estimation methods for turbocharged spark ignition engines," Ph.D. dissertation, Dept. Elect. Eng., Univ. Michigan, Ann Arbor, MI, USA, 2009.

[15] A. Y. Karnik and M. Jankovic, "IMC based wastegate control using a first order model for turbocharged gasoline engine," in Proc. Amer. Control Conf. (ACC), Jun. 2012, pp. 2872-2877.

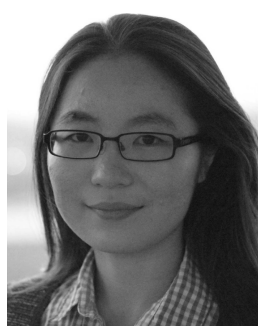

Zeng Qiu received the B.S. degree from the Beijing University of Posts and Telecommunications, Beijing, China, in 2011, and the M.S. degree from the University of Michigan, Ann Arbor, MI, USA, in 2013, where she is currently pursuing the $\mathrm{Ph} . \mathrm{D}$. degree, all in electrical engineering.

Her current research interests include internal model control, adaptive control, and their automotive applications.

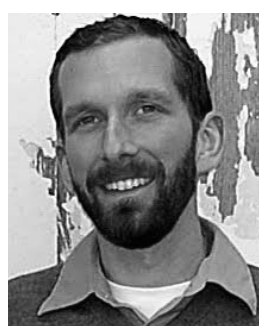

Mario Santillo received the B.S. degree in aeronautical and mechanical engineering from the Rensselaer Polytechnic Institute, Troy, NY, USA, in 2003, and the M.S.E. and Ph.D. degrees in aerospace engineering from the University of Michigan, Ann Arbor, MI, USA, in 2005 and 2009, respectively.

$\mathrm{He}$ is currently with Research and Advanced Engineering, Ford Motor Company, Dearborn, MI, USA. His current research interests include adaptive control and advanced control methods for 


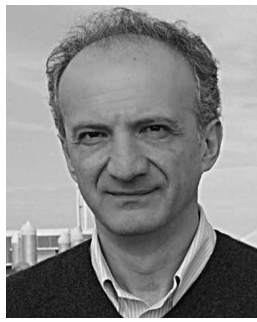

Mrdjan Jankovic (F'04) received the bachelor's degree from the University of Belgrade, Belgrade, Serbia, and the master's and Ph.D. degrees from Washington University, St. Louis, MO, USA.

$\mathrm{He}$ held post-doctoral teaching and research positions with Washington University and the University of California, Santa Barbara, CA, USA. He joined Ford Research, Dearborn, in 1995, where he is currently a Senior Technical Leader, overseeing a team involved in development of powertrain control technologies. He has co-authored one book, four book chapters, and over 100 technical papers. He is a co-inventor of over 50 U.S. patents, 17 of which are implemented in Ford products worldwide.

Dr. Jankovic was a recipient of the IEEE Control Systems Technology Award, two Ford Research Technical Achievement Awards, and three best paper awards from the IEEE, SAE, and AVEC. He served as an Associate Editor of the IEEE TRANSACTIONS ON CONTROL SYSTEMS TECHNOLOGY and the Chair of several IEEE and SAE committees.

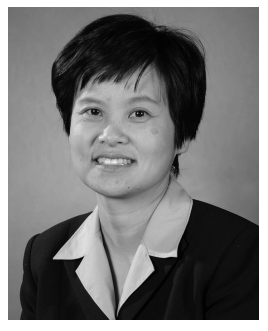

Jing Sun (F'04) received the B.S. and M.S. degrees from the University of Science and Technology of China, Hefei, China, in 1982 and 1984, respectively, and the Ph.D. degree from the University of Southern California, Los Angeles, CA, USA, in 1989.

She was an Assistant Professor with the Department of Electrical and Computer Engineering, Wayne State University, Detroit, MI, USA, from 1989 to 1993. She joined the Ford Research Laboratory, Dearborn, MI, USA, in 1993, where she was involved with the Department of Powertrain Control Systems. After spending almost 10 years in the industry, she came back to academia and joined the faculty of the College of Engineering, University of Michigan, Ann Arbor, MI, USA, in 2003, where she is currently a Professor with the Department of Naval Architecture and Marine Engineering and the Department of Electrical Engineering and Computer Science. She has co-authored a textbook entitled Robust Adaptive Control, and holds 37 U.S. patents. Her current research interests include system and control theory and its applications to marine and automotive propulsion systems.

Prof. Sun is one of the three recipients of the 2003 IEEE Control System Technology Award. 